cleaning urinals. I do not see why negroes should not be subject to extragenital chancres as often as we are. I agree with Dr. Irvine that syphilis should be taught by the dermatologist, who sees these cases early and recognizes them early; furthermore, the subject should be taught in a single department in order to follow up these patients intelligently. Medical schools spend weeks and months in teaching the diagnosis and treatment of syphilis in the late stages, when little can be done; whereas the same amount of time, used in the earlier stages, would be of infinitely more value.

Dr. Jay Frank Schambrrg, Philadelphia: Concerning the comparative value of salvarsan and neosalvarsan, it is very difficult to draw conclusions unless the series of cases was extremely large. One case of syphilis is not like another. The location of the spirochetes in the tissues and their accessibility to medicaments are important factors, influencing the therapeutic results: most of the clinical reports on the comparative value of salvarsan and neosalvarsan are favorable to the former. My own opinion, based on clinical impressions, and on the results of the use of the two drugs in experimental trypanosomiasis, is that salvarsan is very distinctly superior to neosalvarsan.

Dr. Joseph A. Elifott, Ann Arbor, Mich: One of the points we wish to emphasize in this paper is the tendency for late accidents to occur following poor treatment, of which the pill treatment is an excellent example. During the months of October and November, when our supply of salvarsan was very limited, and we were forced to limit the dosage, we had a few accidents, thus showing that even salvarsan, unless given properly and followed by thorough mercurialization, may. prove inefficient. What constitutes efficient treatment depends on the individual case. In all cases, however, we try to give at least five or more injections of salvarsan, followed by prolonged mercurialization either in the form of injections or inunctions.

Dr. John J. Rothwell, New York: In our work we had in mind the thought that Dr. Schamberg expressed just now; we knew that our cases were not all of the same variety, and our chief object in presenting this paper was to stimulate others to take up this work so that a sufficient number of cases might be collected on which to base definite conclusions.

Dr, H. G. Irvinf, Minneapolis: I am not prepared to say that the subject of syphilis should be tatight by one man, but in one department. The dermatologist can call to his assistance men who specialize in other lines, who can discuss special phases of the subject, but the course should be so organized that the student will get a clear idea of the disease as a whole. I do not agree with those who believe that syphilis cannot be controlled. We control tuberculosis and other diseases and why not syphilis? If necessary, the aid of the police could be invoked. They have certainly gone far toward controlling it in Boston.

Chlorosis of Pineapples.-An observation made on pineapples may prove of some value to the human. $M$. O. Johnson of the Hawaiian Experiment Station recently found that the chlorosis of pineapples occurring on highly manganiferous soils can be cured by spraying the leaves with ferrous stiphate. Hence it is a manganese induced chlorosis. There is also a lime induced chlorosis met with in Porto Rico. The lime chlorosis was shown to be due to a lack of iron in the plant, caused by the carbonate of lime diminishing the availability of iron in the soil. At first it was not known whether the chlorosis was due merely to a lack of iron or to a lack of iron combined with a large amount of lime in the plant. Recent work seems to show that it is merely due to a lack of iron. Now the manganese chlorosis may be similar to the lime chlorosis if the manganese acts similarly in merely diminishing the availability of iron in the soil. The recent discovery of Johnson shows that this may be possible. It is possible that the manganese chlorosis is due to a deficiency of iron combined with a direct toxic effect of the manganese. The application of ferrous sulphate to the leaves apparently has a more permanent effect on the manganese plants than on the lime plants. The treatment must be made frequently to maintain the plants in a green and vigorous condition.Science, Dec. 15, 1916, p. 855.

\section{THE SPHENOID SINUS}

\section{A REVIEW OF THE PAST WITH SUGGESTIONS FOR THE FUTURE*}

\section{HANAU W. LOEB, A.M., M.D. ST. LOUIS}

The study of the sphenoid sinus is most fascinating and presents many opportunities. Located as it is on either side of the midline of the under side of the head, often extending far to the opposite side, its size and shape vary with respect to its fellow more than any bilateral organ or cavity of the body. This appears even more remarkable when its relations are considered to such imporiant and fairly constantly placed structures as the cranial cavity, cavernous sinus, internal carotid artery, optic nerve, optic chiasm and pituitary body. The bony coverings of the sinus and the bony outline of other portions of the sphenoid and the adjacent bones give no indication of the extent or shape of the sinus. Even the position of the nasal opening, as I lave shown, is subject to wide variation, while the septum between the two sinuses is conspicuously inconstant in form, size and position.

The operative work done so successfully nowadays in spite of the distance from the anterior nares adds to the interest which we feel for the cavity, while the danger, though slight, of-operating so close to such vital structures as the cavernous sinus, internal carotid arteries and higher brain centers gives it a zest to which only the timid will not respond.

Our understanding of the sphenoid has been of recent growth, but it has developed by leaps and bounds. The remote past contributed but little to this knowledge. The ancients as well as the writers of the middle ages understood the sphenoid sinuses, like the other accessory sinuses of the nose, as being mere accidental cavities with the function of relieving the head of the weight which solid bone would entail. They were, however, considered of great importance by reason of their ability to drain the brain of its humors, or of their disposition to remove pus and other fluid; from the brain cavity. Even Hippocrates suggested that pus in the nose çame in part from the sphenoid sinus, although he did not know the precise pathway it took in its journey.

Schneider's observations, which excluded the nose from any communication with the cranial cavity in life, brought an end to the supposed brain-purging function of the nose and gave rise to speculation as to the purpose of the individual sinuses.

The sphenoid sinus took but little part in the hypotheses that were advanced, either from the fact that little was known as to its extent and relation to the nose, or because no explanation, reasonable or unreasonable, could be given for its existence. It is quite obvious, therefore, why so little attention was paid to it for all these years and why, for instance, Hyrt in 1882 stated that the sphenoid sinus was entirely beyond the range of manual and instrumental attack.

But the wonderful work of Zuckerlandl published the same year was destined to change this entire conception of the subject, for he gave the study of the normal and pathologic anatomy of the accessory sinuses of the nose an impetus which it retains even to this day. His findings as to the sphenoid are particularly of value

* Read before the Section on Laryngology, Otology and Rhinology at the Sixty-Seventh Annual Session of the American Medical Associatiun, Detroit, June, 1916. 
in that he established the usual relation of the sinus to the optic nerve. Very little has been added to this work. We may mention Onodi, who demonstrated the relation of the sphenoid and posterior ethmoidal cells, the writer who showed the variation in size and shape and the vulnerability of the optic nerve when the sinus opening is high in the upper third of the anterior wall; and Sluder, who made extensive studies on the relation of the sinus to the various cranial nerves.

The investigations of Zuckerkandl were soon followed by determined efforts to utilize them for therapeutic purposes, although it was some time before effective measures were adopted. The first article in which the sphenoid sints is mentioned in the caption appeared in 1886, by Berger and Tyrman. ${ }^{1}$ Even then they supposed that the suppuration was always due to syphilis, scrofula or traumatism.

Schaeffer, who was the first to open the sphenoid, in 1885 contributed a great deal to the development of the knowledge of this cavity, while Berger and Tyrman, Rolland, Heryng, Rualt, Quénu, Clark, Cozzolino and others wrote more or less extensively during the first ten years following the publication of Zuckerkandl's monograph. Schaeffer and his followers endeavored to secure drainage of the sinus by simply enlarging the natural opening, although Schaeffer himself attempted to extend the enlargement to the floor of the cavity. Killian in 1900 called attention to the frequency of the coincident involvement of the last posterior ethmoid cell and the possibility of securing a larger opening by operating through the pars ethmoidalis. However, it was Hajek who demonstrated by his painstaking investigation and observations that the best results could be obtained only by a resection of both the pars ethmoidalis and the pars nasalis of the anterior wall of the sinus. Since these publications little has been done except to confirm them and to establish a somewhat more definite indication for the operation.

The operative attack of the hypophysis by way of the sphenoid sinus, which has been developed during the past few years, has been accepted as the most satistactory plan, particularly when removal of the bony wall alone is required. The technic is easy for experienced operators on the nose and the results are sufficiently good to justify the interest that has been manifested in it. The development of roentgenographic studies in connection with the pituitary question has been satisfactory, though it must be admitted that roentgenography has so far been of little value in the diagnosis of sphenoid empyema.

We have thus seen that the entire progressive part of our knowledge of the sphenoid sinus has occurred within the past thirty-five years, while its surgery was practically an unknown quantity thirty years ago.

We are very apt to be filled with pride when we contemplate the development of a new line of work which has achieved as nuch as that of the sphenoid in such a short time, particularly in anatomy, diagnosis and surgery. In order that we may not feel too well satisfied or despair that everything has already been discovered, it may not be amiss to view some of the problems which remain unsolved in connection fvith this subject.

1. Among the very first that should attract our attention is the cause of the excavation of the sphenoid body, resulting in the formation of these sinuses, with such a variety in shape and size. The pressure of the air in the nose in breathing might appear to be the

\footnotetext{
1. Berger and Tyrman: Centralbl. f. Laryngol., 1886.
}

explanation, especially as the changes take place during the air-breathing stage of the individual. So far experiments with this in view have added nothing toward its solution; there is still plenty of opportunity in this direction.

2. Associated with this question is that of the relation of the posterior ethmoid cells to the sphenoid. Why does the last posterior ethmoid cell sometimes project itself into the sphenoid and replace the corresponding sphenoid sinus? I have shown that this occurred twice in the thirty sphenoid sinuses studied by me, and that in those instances the optic nerve ran for a considerable distance along the external wall of this ethmoid cell instead of having only the slightest relation at the postero-external angle of the cell. Is my surmise correct that under the former circumstances the optic nerve is more vulnerable to ethmoid infections?

3. What bearing has the sphenoid sinus on the cranial nerves in its neighborhood? Are they susceptible to the influence of purulent infections of the sinus?

4. The physiology of the sphenoid sinus is still almost an unknown quantity. Beyond its value as a means of decreasing the weight of the head without reducing the bone surface area, we have little to say, and this was held nearly 3,000 years ago. By the development of the sphenoid sinus from the nasal cavity there is a consequent increase in the respiratory mucous membrane. When shall we be able to answer the question as to what may be the purpose of this great extension of the nasal mucosa?

5. As to etiology, we are still much in the dark. We know that suppuration of the sphenoid may follow certain of the infectious diseases; we know it frequently follows acute coryza and suppuration in other sinuses; we know many varieties of pathogenic bacteria find their way into the cavity, but we do not know what selective agent accounts for the attack of the sinus at the specified time. We do not know why the sinus should escape infection when every necessary condition is present so far as our knowledge goes; at any rate the opportunity is abundant, the attack rare. No one needs, therefore, complain that etiologic problems are wanting.

6 . The symptomatology of sphenoid sinus suppuration presents a wide range. In many cases practically no subjective signs whatever are present; in others the suffering is most acute. While this may be accounted for in part by the variation of the drainage, the explanation does not suffice for cases in which the symptoms fail to agree with the facility of drainage. We hear of an attack of blindness ascribed to sphenoid suppura. tion and relieved by appropriate treatment addressed to the sphenoid sinus, and we forthwith look to it as an explanation of eye symptoms of any character, provided they have no intra-ocular cause. We read of a case of acute mania due to suppuration of this cavity and we rush to tap the sinus in all cases of mania, whether there is suppuration or not.

What we need is to have some one determine the symptom basis so that a clearer line may be drawn between the symptoms due to the sphenoid and those which have some other origin.

7. Our best agents for diagnosis are cocain, the Killian speculum and the Holmes nasopharyngoscope. Roentgenography offers but little; we can make out the lateral bony boundaries of the sinus fairly well, particularly when we take advantage of stereoscopy. We can see it in the outline in some measure by an anterion 
view picture, but on account of its distance from the plate, we cannot determine the presence of pus, as in the case of frontal and maxillary sinuses. Who will find the solution for this problem?

8. We have not achieved the highest point in the surgery of the sphenoid. Notwithstanding the ease with which we resect the anterior wall, we still must submit to failures, even though many operations are followed by marvelous results. The suppuration continues in spite of our most earnest efforts. On the other hand, patients treated expectantly sometimes recover. Success, we may say, now depends largely on the judgment of the operator. Will not some earnest investigator place this on a basis more independent of the mere judgment of the operator than now obtains?

9. Who will so classify the chain of nervous symptoms, headache, etc., that sphenoid operations for their relief may be rational and not experimental? Truly there is a large field here for study and investigation.

10. We cannot fully account for postoperative accidents and results by what we know, nor are they always due to operative inefficiency. We need a great deal more light in this regard.

On the whole, it is manifest that we have not reached the utmost limits of knowledge by what we have accomplished in the study of the sphenoid sinus. For that reason $I$ have endeavored to show, not how little we know, but how much we are to know.

\section{THE SURGICAL TREATMENT OF CHRONIC MAXILLARY SINUSITIS *}

\author{
WILLIAM E. SAUER, M.D. \\ ST. LOUIS
}

According to Jonathan Wright, Galen was the first to mention the maxillary sinus, but Brengar was the first to describe it. However, the first description of any real value was made by Highmore in 1651 and since then the cavity has borne his name. At the same time he reported a case of suppurative disease of the sinus. Mollinette in 1675 made a crucial incision on the jaw, and with a trephine penetrated the antrum and located an abscess. Meibomits, who died in 1655, invented the operation now known as the Cooper operation. Cooper described his operation in 1717. Jourdain, a Paris dentist, in 1765 reported a method of washing out the antrum through the natural opening. Grooch, an English surgeon, who died in 1780, and John Hunter in his treatise on the human teeth proposed perforating the antrum through its nasal surface. Wright states that in going over the history of the antrum and its diseases one will perceive that all recent procedures have been long anticipated.

The treatment of maxillary sinus diseases was apparently neglected; at least it did not receive the attention it had formerly. It received a new impetus in 1886 when Ziem pointed out that disease of this cavity was not only far from infrequent, but a common occurrence. Since then a number of operative measures have been devised.

The operations for chronic maxillary sinus disease may be divided into three classes: (1) those in which the cavity is attacked only through the mouth, as in the Cooper and Kuster operations ; (2) those in which

* Read before the Section on Laryngology, Otology and Rhinology "Read before the Section on Laryngology, Otology and Rhinology tion, Detroit, June, 1916. the cavity is attacked only through the nose, as in the Krause, Mikulicz, Canfield and Skillern operations; and (3) those in which the antrum is attacked through the oral and nasal cavities, as in the Caldwell-Luc and Denker operations.

The Cooper operation is employed only when a carious tooth or root is the etiologic factor; usually the second bicuspid or the first and the second molar are removed, the opening enlarged and smoothed with a drill, and drainage established by means of gauze or a specially devised tube or obturator, which is worn by the patient until the cavity is healed. The chief disadvantage is that if there are any marked pathologic changes in the sinus the process will not get well.

The Kuster-Desault operation consists of a free opening of the antrum through the canine fossa and curetting the walls of the cavity. This operation has been practically abandoned.

Of the intranasal operations, the simplest is the Krause-Mikulicz, which consists in the removal of the anterior third of the lower turbinate. 'The antrum is then punctured with a large trochar and thoroughly irrigated. The opening is enlarged by means of a punch forceps and packed with gauze. This packing is removed at the end of twenty-four hours. The patient can be taught to irrigate the cavity until healing has been secured. The advantage is its simplicity. The disadvantage is that if any marked pathologic changes are present, it will not suffice.

The Canfield operation, which is a modification of the Denker operation, to be described later, consists of the intranasal, submucous resection of the naso-antral wall under local anesthesia. A 1 per cent. solution of cocain is injected in front of the inferior turbinate; the needle is directed so that the solution is carried under the periosteum in the canine fossa. The lower turbinate and lateral wall of the nose are cocainized in the usual way. An incision is then made from the middle of the anterior attachment of the inferior turbinate down to the floor of the nose. This incision is made through all the tissues down to the bone. A small elevator is introduced and the periosteum elevated from the crista pyriformis externally toward the canine fossa, and internally toward the nose, including the mucous membrane covering the inferior turbinate and the lateral wall of the inferior meatus. The bony structure of the inferior turbinate is removed, and the antrum may then be opened by means of a chisel, trephine or rongeur bone forceps. Enough of the bone in the canine fossa, as well as the bony wall of the inferior meatus as far back as the posterior wall of the antrum, is resected. A large-sized ear speculum is introduced and the interior of the antrum is inspected. A flap of mucous membrane is turned into the antrum from the nose and the cavity is packed with gauze, which is allowed to remain from twenty-four to forty-eight hours. The after-treatment consists of touching subsequent granulation tissue with caustics. Healing occurs in from ten days to three months. The advantages of the operation are that there is very little disturbance following the operation; the sinus can always be inspected, and the process of healing noted; drainage is at the lowest possible point. The objection to the operation is the removal of the inferior turbinate.

Skillern has modified the Canfield operation in that, after cocainization, a perpendicular incision is made slightly in front of and above the anterior end of the inferior turbinate extending well into the floor of the 